\title{
Endarterectomía carotídea en octogenarios: comparación de desenlaces con pacientes menores de 80 años, experiencia en la Fundación Cardio Infantil
}

\author{
Fernando Monroy Melo ${ }^{1}$, Katerine Andrea Cepeda Páez ${ }^{2}$, \\ Jaime Camacho Mackenzie ${ }^{3}$, Carlos Eduardo Pinzón Flórez ${ }^{4}$
}

\section{Resumen}

Introducción. Dado el incremento de los pacientes con edades superiores a los 80 años que son llevados a cirugía cada año en nuestra institución, consideramos importante conocer el comportamiento en términos de morbilidad y mortalidad tempranas de los pacientes octogenarios sometidos a endarterectomía carotídea.

Objetivo. Evaluar los resultados a 30 días de pacientes octogenarios con enfermedad carotídea grave, sintomática y asintomática, sometidos a endarterectomía carotídea, comparados con una cohorte histórica de pacientes de menor edad operados desde 1995 en la Fundación Cardio Infantil. Se registraron como variables dependientes la mortalidad y el accidente cerebrovascular.

Resultados. Se registraron 218 endarterectomías carotídeas, $189(86,6 \%)$ en pacientes menores de 80 años y $30(13,4 \%)$ en pacientes octogenarios. En pacientes octogenarios no se presentaron accidentes cerebrovasculares $(0 \%)$ y la mortalidad fue de $3,3 \%$.. En el grupo de pacientes menores de 80 años se presentaron accidentes cerebrovasculares $(1,7 \%)$ y muerte $(1,1 \%)$.

1 Médico, residente de Cirugía Vascular y Angiología, Fundación Cardio Infantil, Universidad del Rosario, Bogotá, D.C., Colombia.

2 Médica asociada, Departamento de Investigación, Departamento de Cirugía Vascular y Angioloía. Fundación Cardio Infantil, Bogotá, D.C., Colombia.

3 Médico cirujano, Departamento de Cirugía Vascular, Fundación Cardio Infantil - Instituto de Cardiología, Bogotá, D.C., Colombia.

4 Médico, Centro de Investigaciones Clínicas, Universidad de Nuestra Señora del Rosario, Bogotá, D.C., Colombia. 
Conclusiones. Los pacientes octogenarios con adecuada funcionalidad y sin contraindicaciones claras pueden ser sometidos a endarterectomía carotídea de manera segura.

Palabras clave: endarterectomía carotídea, morbilidad, accidente cerebrovascular.

\section{Title}

Carotid endarterectomy in octogenarians: comparison of outcomes in younger patients, the Fundación Cardio Infantil experience.

\begin{abstract}
Background: Given the increase in patients over 80 years old who are taken to surgery each year in our institution we considered important to know the behavior in terms of early morbidity and mortality of those taken to carotid endarterectomy.
\end{abstract}

Objective: To assess outcomes at 30 days in octogenarians with symptomatic and asymptomatic severe carotid disease taken to carotid endarterectomy compared to a historic cohort of younger patients operated since 1995 at Fundación Cardio Infantil. End points were mortality and stroke.

Results: A total of 218 carotid endarterectomies, $189(86,6 \%)$ in patients under 80 years and 30 $(13,4 \%)$ in octogenarians. Octogenarias had 3,3\% cerebrovascular events and $0 \%$ death. The group of patients less than 80 years had $1,7 \%$ cerebrovascular events and $1,1 \%$ death.

Conclusions: Octogenarians with proper functionality and no clear contraindications can be carried safely to carotid endarterectomy.

Key words: carotid endarterectomy, stroke, morbidity.

\section{Introducción}

La enfermedad carotídea con estenosis crítica tiene indicaciones quirúrgicas claras tanto para pacientes sintomáticos como asintomáticos[1-3]. En estudios anteriores se han demostrado los beneficios de la cirugía en pacientes con estenosis significativa (mayor de $60 \%$ ), convirtiéndose en el método más eficaz para lograr una disminución en la probabilidad de presentar un accidente cerebrovascular a corto y a largo plazo[4-7].

La expectativa de vida en nuestro país ha aumentado en los últimos 25 años. La población de pacientes mayores de 80 años ha aumentado en $400 \%$, aproximadamente, con 489.200 personas en el último censo en nuestro país en 2005[8]. La incidencia de enfermedad carotídea en mayores de 80 años oscila entre $7 \%$ y $12 \%$ en las series norteamericanas[9]. En un estudio realizado por Hamel et al. en hospitales especializados, se observó un aumento de los procedimientos mayores y menores en pacientes que superan los 80 años de edad, con múltiples enfermedades concomitantes y resultados comparables a los de grupos de pacientes de menor edad[10, 11]. En general, la mortalidad y la morbilidad de los pacientes octogenarios sometidos a cirugía mayor son mayores que las de la población general[12]. Se han reportado resultados excelentes en procedimientos mayores y pacientes octogenarios, en otros tipos de cirugía[13, 14].

Aunque la prevalencia de la enfermedad aumenta con la edad, muchos estudios prospectivos y de asignación 
aleatoria han excluido los pacientes de 80 años o mayores, porque usualmente se consideran como de "alto riesgo" para endarterectomía carotídea. En la mayoría de las series grandes, se considera a la edad avanzada (80 años o más) como factor de alto riesgo para la endarterectomía carotídea y se ha utilizado como indicación para procedimientos alternos, como el manejo médico, la angioplastia y la colocación de stents[15-17].

Dado el incremento de los pacientes con edades superiores a los 80 años que son sometidos a cirugía cada año en nuestra institución, consideramos importante conocer el comportamiento en términos de morbilidad y mortalidad tempranas de los pacientes octogenarios sometidos a endarterectomía carotídea. Los avances recientes en los tratamientos endovasculares han llevado a proponer la angioplastia carotídea y el stent como tratamiento inicial para estos pacientes llamados de "alto riesgo"[18]. Un estudio realizado por Stoner et al. de manera prospectiva y otros estudios realizados por Timaran et al. y Sidawy et al. de manera retrospectiva, muestran reportes de series con tasas de morbilidad y mortalidad perioperatoria en endarterectomía carotídea realmente bajas y comparables con las estadísticas presentadas en grupos de menor edad[19-23].

\section{Materiales y métodos}

El diseño del estudio fue observacional analítico, y de tipo de casos y controles con casos prevalentes. Se tomó como cohorte subyacente una cohorte histórica consecutiva de pacientes sometidos a endarterectomía carotídea. Se revisaron los registros de todos los pacientes llevados a endarterectomía carotídea, sintomáticos y asintomáticos, de manera retrospectiva durante 15 años desde enero de 1995 hasta enero de 2010 en la Fundación Cardio Infantil. Se excluyeron todos a los que se les practicó algún tipo de cirugía concomitante con la endarterectomía carotídea (por ejemplo, revascularización miocárdica). Se hizo la búsqueda de la información en la historia clínica (notas de evolución, métodos diagnósticos, registros anestésicos y descripciones quirúrgicas) y se registraron las variables independientes (tabla 1) y las variables dependientes (tabla 4). Se registraron, además, detalles quirúrgicos (tabla 3). Se anotaron los datos provenientes de los estudios del laboratorio vascular y valoración prequirúrgico (tabla 2). El grado estimado de estenosis se determinó teniendo en cuenta los parámetros establecidos para el laboratorio vascular por la Universidad de Washington.

Se establecieron los dos grupos de estudio, clasificándolos exclusivamente por la edad: el grupo 1, o de control, en el que se incluyeron pacientes menores de 80 años y, el grupo 2 de pacientes octogenarios, grupo de casos.

Por protocolo de la institución, todos los pacientes son monitorizados en la 
unidad de cuidados intensivos durante 24 horas, con estricto control de la presión arterial y análisis neurológico.

Los objetivos principales medidos fueron: muerte, infarto agudo del miocardio y accidente cerebrovascular perioperatorio. Los objetivos secundarios se centraron en la medición del accidente isquémico transitorio, lesión de nervio periférico, tiempo hospitalización en la unidad de cuidados intensivos y tiempo de hospitalización en salas.

\section{Variables}

Se creó una base de datos con 47 variables, de acuerdo con las definiciones estándar, incluyendo variables demográficas, antecedentes patológicos, estudios imagenológicos, características del procedimiento, y resultados de mortalidad y episodios neurológicos.

\section{Sesgos}

Se definieron adecuadamente los criterios de inclusión y exclusión para controlar el sesgo de selección. El sesgo de información de control estandarizando las definiciones de cada variable y el entrenamiento de los recolectores; un solo digitador entrenado y la revisión de la base de datos con auditoría de historias no concordantes. El sesgo de mala clasificación no diferencial se controló evaluando el proceso de determinación de los desenlaces y los criterios de inclusión.

\section{Tamaño de muestra}

Se tomó toda la población de pacientes operados en la institución que fueron sometidos a endarterectomía carotídea exclusivamente.

\section{Métodos estadísticos}

Se hizo una descripción general de cada variable mediante análisis univariado; las variables continuas se expresaron como media \pm desviación estándar, o medianas con rango máximo y mínimo, para medidas con distribución normal y sin ella, respectivamente. Las variables categóricas se expresaron en frecuencia absoluta y relativa.

Para la comparación de los grupos, las variables continuas fueron evaluadas usando la prueba de t de Student o MannWhitney U, y las variables categóricas, con el test de Fisher o del chi cuadrado.

\section{Resultados}

Se practicaron 218 endarterectomías carotídeas en 189 pacientes, en el periodo comprendido entre enero de 1995 y enero de 2010. Se realizaron 188 procedimientos (grupo 1) en pacientes menores de 80 años y 30 procedimientos (grupo 2) en pacientes octogenarios, correspondientes a $86,3 \%$ y a $13,7 \%$, respectivamente. Las características demográficas de los pacientes se muestran en la tabla 1. 
Tabla 1

Antecedentes

\begin{tabular}{lccc}
\hline & $\begin{array}{c}\text { Grupo 1 } \\
\mathrm{n}(\%)(\mathrm{n}=188)\end{array}$ & $\begin{array}{c}\text { Grupo 2 } \\
\mathrm{n}(\%)(\mathrm{n}=30)\end{array}$ & $\mathrm{P}$ \\
\hline Edad, mediana (mínima-máxima) & $70(38-79)$ & $82(80-89)$ & \\
Hombres & $118(62,8)$ & $20(66,7)$ & 0,68 \\
Hipertensión & $164(87,2)$ & $25(83,3)$ & 0,56 \\
Diabetes mellitus & $39(20,7)$ & $2(6,7)$ & 0,07 \\
EPOC & $20(10,6)$ & $5(16,7)$ & 0,34 \\
Tabaquismo & $107(56,9)$ & $17(56,7)$ & 0,98 \\
ICC & $14(7,4)$ & $3(10)$ & 0,63 \\
IRC & $11(5,8)$ & $2(6,7)$ & 0,86 \\
Enfermedad coronaria & $87(46,5)$ & $9(30)$ & 0,09 \\
EAOC & $23(12,2)$ & $2(6,7)$ & 0,37 \\
Medicamentos control factores de riesgo & $174(92,6)$ & $25(83,3)$ & 0,09 \\
Evaluación neurológica & & & 0,02 \\
Sintomáticos & $110(58,5)$ & $25(83,7)$ & \\
Asintomáticos & $78(41,5)$ & $5(16,3)$ & \\
\hline
\end{tabular}

EPOC: enfermedad pulmonar obstructiva crónica; ICC: insuficiencia cardiaca congestiva; IRC: insuficiencia renal crónica; EAOC: enfermedad arterial oclusiva crónica; medicamentos control de factores de riesgo: ácido acetilsalicílico y estatinas.

El porcentaje de procedimientos en hombres en el grupo 1 fue de $62,8 \% \mathrm{y}$, en el grupo 2, de 66,7\%. No se encontraron diferencias estadísticamente significativas en la mayoría de las variables demográficas, salvo en la incidencia de diabetes mellitus y enfermedad coronaria, entre los dos grupos. Se observa una diferencia significativa en la presencia de síntomas neurológicos entre los dos grupos, con $\mathrm{p}$ de 0,01 , dada principalmente por la menor cantidad de asintomáticos entre los octogenarios.

No se encontraron variables estadísticamente significativas en la valoración prequirúrgica y los grados de estenosis entre los dos grupos (tabla 2). 
Tabla 2

Evaluación preoperatoria

\begin{tabular}{|c|c|c|c|}
\hline & $\begin{array}{c}\text { Menores de } 80 \text { años } \\
\mathrm{n}(\%)(\mathrm{n}=188)\end{array}$ & $\begin{array}{l}\text { Octogenarios } \\
\mathrm{n}(\%)(\mathrm{n}=30)\end{array}$ & $\mathrm{P}$ \\
\hline NYHA & & & 0,71 \\
\hline I & $35(18,6)$ & $4(13,3)$ & \\
\hline II & $134(71,3)$ & $22(73,3)$ & \\
\hline III & $19(10,1)$ & $4(13,3)$ & \\
\hline ASA & & & 0,33 \\
\hline I & $24(12,8)$ & $2(6,7)$ & \\
\hline II & $81(43,1)$ & $17(55,7)$ & \\
\hline III & $83(44,1)$ & $11(36,7)$ & \\
\hline Grado de estenosis derecha & & & 0,35 \\
\hline$<19$ & $65(34,6)$ & $7(23,3)$ & \\
\hline $20-49$ & $3(1,6)$ & $0(0,0)$ & \\
\hline $50-79$ & $40(21,3)$ & $11(36,7)$ & \\
\hline $80-99$ & $78(41,5)$ & $12(40)$ & \\
\hline 100 & $2(1,1)$ & $0(0,0)$ & \\
\hline Porcentaje de estenosis derecha & $80(40-100)$ & 79,5 (19-99) & 0,6 \\
\hline Grado de estenosis izquierda & & & 0,01 \\
\hline$<19$ & $47(25)$ & $9(30)$ & \\
\hline $20-49$ & $8(4,6)$ & $2(6,7)$ & \\
\hline $50-79$ & $47(25)$ & $12(40)$ & \\
\hline $80-99$ & $86(45,7)$ & $6(20)$ & \\
\hline 100 & $0(0,0)$ & $1(3,3)$ & \\
\hline Porcentaje de estenosis izquierda & $80(19-99)$ & $77,5(19-100)$ & 0,03 \\
\hline
\end{tabular}

NYHA: Clasificación de la New York Heart Association; ASA: American Society of Anesthesiology.

Los detalles de los procedimientos quirúrgicos fueron similares, con una diferencia estadísticamente significativa que fue encontrada en la cantidad de pacientes con colocación de parche. En
$58,3 \%$ de los pacientes del grupo 1 se utilizó parche sintético de dacrón y, en $4,7 \%$, parche venoso; en $93,3 \%$ de los pacientes del grupo 2 se usó parche de dacrón (tabla 3). 
Tabla 3

Características operatorias

\begin{tabular}{lccc}
\hline & $\begin{array}{c}\text { Menores de } 80 \text { años } \\
\mathrm{n}(\%)(\mathrm{n}=188)\end{array}$ & $\begin{array}{c}\text { Octogenarios } \\
\mathrm{n}(\%)(\mathrm{n}=30)\end{array}$ & $\mathrm{P}$ \\
\hline Lado operado & & & 0,49 \\
Izquierdo & $94(50)$ & $13(43,3)$ & \\
Derecho & $94(50)$ & $17(55,7)$ & 0,1 \\
Shunt & $129(68,6)$ & $25(83,3)$ & 0,006 \\
Parche & $121(64,4)$ & $28(93,3)$ & 0,42 \\
Tipo de cirugía & & $29(100)$ & \\
Convencional & $184(97,9)$ & $0(0,0)$ & \\
Eversión & $4(2,1)$ & & \\
\hline
\end{tabular}

Los objetivos del estudio se discriminaron según la clasificación neurológica, teniendo en cuenta los lineamientos expuestos por los estudios más significativos en el área, como lo son el ACAS (Endarterectomy for Asymptomatic Carotid Artery Stenosis)[24] y el NASCET (North American Symptomatic Carotid Endarterectomy Trial)[25]. En estos estudios, los episodios neurológicos se estandarizaron como accidentes cerebrovasculares, los que tenían secuelas de más de 24 horas y, como accidente isquémico transitorio, los de más de 25 segundos de duración sin secuelas mayores a las 24 horas. No se estableció una permisividad de accidente isquémico transitorio para pacientes sometidos a endarterectomía carotídea. Consideramos que éste es un factor importante, dado que en nuestro estudio existe una diferencia estadísticamente significativa en la incidencia de accidente isquémico transitorio en pacientes octogenarios.

Encontramos, también, que la lesión del nervio hipogloso también era más frecuente en pacientes octogenarios, con una diferencia estadísticamente significativa; no así para las lesiones de la rama marginal del nervio facial y del laríngeo recurrente.

La mortalidad perioperatoria global fue de $1,1 \%$ en procedimientos del grupo 1 y de $3,3 \%$ en el grupo 2 . En el grupo 2 no se presentaron accidentes cerebrovasculares y, en el grupo 1, se presentaron en $1,1 \%$, sin diferencias estadísticamente significativas (tabla 4). 
Tabla 4

Desenlaces

\begin{tabular}{lccc}
\hline & $\begin{array}{c}\text { Menores de } 80 \text { años } \\
\mathrm{n}(\%)(\mathrm{n}=188)\end{array}$ & $\begin{array}{c}\text { Octogenarios } \\
\mathrm{n}(\%)(\mathrm{n}=30)\end{array}$ & $\mathrm{P}$ \\
\hline Hematoma & $8(4,3)$ & $1(3,3)$ & 0,83 \\
Infección & $1(0.5)$ & $1(3,3)$ & 0,13 \\
Nervio hipogloso & $2(1,1)$ & $3(10)$ & 0,002 \\
Nervio laríngeo & $8(4,3)$ & $3(10)$ & 0,18 \\
Nervio facial & $8(4,3)$ & $2(6,7)$ & 0,55 \\
AIT & $3(1,6)$ & $3(10)$ & 0,009 \\
IAM & $2(1,1)$ & $0(0,0)$ & 0,57 \\
ACV menor de 30 días & $2(1,1)$ & $0(0,0)$ & 0,57 \\
ACV contralateral & $0(0,0)$ & $0(0,0)$ & - \\
Muerte & $2(1,1)$ & $1(3,3)$ & 0,32 \\
\hline
\end{tabular}

AIT: accidente isquémico transitorio; IAM: infarto agudo del miocardio; ACV: accidente cerebrovascular.

En la tabla 5 se describe la aparición de 30 días en los grupos, estratificados de enfermedad cerebrovascular menor en sintomáticos y asintomáticos.

Tabla 5

Aparición de enfermedad cerebrovascular menor de 30 días

\begin{tabular}{lcc}
\hline & $\begin{array}{c}\text { Control } \\
\mathrm{n}=188\end{array}$ & $\begin{array}{c}\text { Octogenarios } \\
\mathrm{n}=30\end{array}$ \\
\hline $\begin{array}{l}\text { Sintomáticos } \\
\text { Asintomáticos }\end{array}$ & $\begin{array}{l}1(0,91 \%) \\
1(1,3 \%)\end{array}$ & $0(0,0)$ \\
\hline Total & $2(1,7 \%)$ & $0(0,0)$ \\
\hline
\end{tabular}

El promedio de estancia hospitalaria fue similar. No se encontraron diferencias estadísticamente significativas entre los grupos (tabla 6). 
Tabla 6

Estancia por subgrupos

\begin{tabular}{|c|c|c|c|c|}
\hline \multirow{2}{*}{$\begin{array}{l}\text { Estancia días mediana } \\
\text { (RIQ) }\end{array}$} & \multicolumn{2}{|c|}{ Control $n=188$} & \multicolumn{2}{|c|}{ Octogenarios $n=30$} \\
\hline & Sintomáticos & Asintomáticos & Sintomáticos & Asintomáticos \\
\hline UCI & 1 & $1(1-2)$ & $2(1-2)$ & $2(1-2)$ \\
\hline Salas & $1(1-2)$ & 1 & $1(1-2)$ & 1 \\
\hline Total & $2(2-3)$ & 2 & $3(2-4)$ & $3(2-4)$ \\
\hline
\end{tabular}

UCI: unidad de cuidados intensivos; RIQ: rango intercuartil, $\mathrm{p}=0,6$.

En las complicaciones menores, como hematoma de la herida quirúrgica e infección, no se encontró diferencia estadísticamente significativa. Las lesiones nerviosas que comprometían el nervio laríngeo recurrente y la rama marginal del nervio facial no presentaron diferencias significativas. Hubo lesión del nervio hipogloso en $10 \%$ de los pacientes del grupo 2.

\section{Discusión}

Los pacientes octogenarios pueden tener resultados de mortalidad y enfermedad cerebrovascular similares a los de los pacientes jóvenes sometidos a endarterectomía carotídea. Un estudio realizado por Kang y Chung, en el Massachusetts General Hospital (Harvard Medical School), demostró que las tasas de mortalidad y morbilidad perioperatoria en pacientes octogenarios son similares a las de pacientes menores de 80 años[18]. En otros estudios anteriores a éstos, se compararon las características y los desenlaces de los pacientes mayores y menores de 80 años sometidos a endarterectomía carotídea y se encontró que eran muy similares, a excepción de la edad[19, 26-29]. Los resultados de estos estudios con respecto a la morbilidad asociada y mortalidad perioperatorias no son estadísticamente significativos, lo que concuerda con nuestros hallazgos. Es de anotar que estos hallazgos son el reflejo del trabajo en instituciones de referencia e instituciones universitarias. Estas observaciones contrastan con las encontradas por Fisher et al.[30] y Hsia et al.[31], quienes examinaron los datos del Medicare sobre la morbilidad y mortalidad de pacientes mayores, y encontraron que la morbimortalidad era mayor en hospitales comunitarios y en hospitales donde estos procedimientos son llevados a cabo de manera esporádica y realizados por cirujanos que practican estos procedimientos pocas veces al año. Aunque estos datos no reflejan lo que sucede en nuestro país, creemos que pueden sugerir la realidad nacional. 
El hecho de que la endarterectomía carotídea se pueda llevar a cabo de manera segura en pacientes octogenarios, no significa que debería realizarse en todos estos pacientes. El riesgo de base de presentar efectos adversos a medida que avanza la edad también se incrementa, así como la probabilidad de necesitar manejo multidisciplinario[18-19]. Se hace necesario, definir la utilidad de un procedimiento seguro y efectivo en los pacientes octogenarios.

Los hallazgos en pacientes octogenarios son comparables con los de los pacientes más jóvenes. La endarterectomía carotídea en octogenarios es ahora más frecuente (tabla 7), no sólo por el aumento de la población sino por la experiencia adquirida por el equipo quirúrgico que involucra tanto a cirujanos como anestesiólogos y, en general, a todas las personas incluidas en el manejo de estos pacientes. También, encontramos que a través de los años las variaciones en la técnica quirúrgica han venido disminuyendo, para terminar en una estandarización de la técnica quirúrgica que hace que los procedimientos sean más seguros.

\section{Tabla 7}

Frecuencia de efectos adversos en diferentes periodos

\begin{tabular}{cc}
\hline $\begin{array}{c}\text { Rango } \\
\text { de fechas }\end{array}$ & $\begin{array}{c}\text { Pacientes octogenarios } \\
\text { sometidos a cirugía }\end{array}$ \\
\hline $1995-1998$ & 2 \\
$1999-2004$ & 9 \\
$2005-2010$ & 19 \\
\hline
\end{tabular}

Lo registrado anteriormente demuestra la necesidad de llevar a cabo estudios prospectivos de asignación aleatoria, con el objetivo de determinar la utilidad de la endarterectomía carotídea en estos pacientes. Consideramos que definir la endarterectomía carotídea como un procedimiento seguro para pacientes octogenarios es un gran paso para el lineamiento de estos estudios, necesarios para la evaluación de la utilidad. En el estudio realizado por Cronenwett et al.[32] se hace un análisis del costo y el beneficio de la endarterectomía carotídea para pacientes asintomáticos, y se demuestra que la edad es el factor determinante más importante dada su capacidad de influir en la expectativa de vida del paciente. Esta relación ha sido puesta a prueba, pues con el paso del tiempo estamos tratando pacientes de mayor edad con funciones intelectuales y físicas sorprendentemente buenas.

En este mismo estudio se considera que, aproximadamente, $50 \%$ de 1 os pacientes son sometidos a angiografía, la cual aumenta la morbilidad asociada y el costo de tratar a estos pacientes. La estandarización de los laboratorios vasculares ha disminuido de manera drástica la utilización de la angiografía como método diagnóstico, y ha permitido obtener buenos resultados de correlación quirúrgica[33, 34]. En nuestra institución utilizamos el dúplex carotídeo como el único examen para diagnóstico y clasificación, en casi todos los casos. 
En conclusión, los pacientes octogenarios pueden ser sometidos a endarterectomía carotídea de manera segura. Consideramos que la selección de los pacientes es un factor importante a la hora de definir candidatos a cirugía, lo cual concuerda con nuestro estudio al demostrar que no existían diferencias significativas entre los dos grupos. Se hace necesario hacer un seguimiento a largo plazo para definir la utilidad del procedimiento en pacientes octogenarios.

\section{Agradecimientos}

Agradecemos a la Fundación Cardio Infantil, por el apoyo y el acceso a la información para el desarrollo de este estudio.

\section{Conflicto de interés}

No declarados.

\section{Bibliografía}

1. ACAS collaborators. Endarterectomy for asymptomatic carotid artery stenosis. Executive Committee for the Asymptomatic Carotid Atherosclerosis Study. JAMA. 1995;273:1421-8.

2. NASCET collaborators. Beneficial effect of carotid endarterectomy in symptomatic patients with high-grade carotid stenosis. North American Symptomatic Carotid Endarterectomy Trial Collaborators. $N$ Engl J Med. 1991;325:445-53.
3. Hallyday A, Mansfield A, Peto R, Marro $\mathrm{C}$, Thomas D, Peto C et al. Prevention of disabling and fatal strokes by successful carotid endarterectomy in patients without recent neurological symptoms: Randomized controlled trail. Lancet. 2004;363:1491-502.

4. Farrel B, Fraser A, Sandercock P, Slattery J, Warlow CP. Randomized trial of endarterectomy for recently symptomatic carotid stenosis: Final results of the MRC European Carotid Surgery Trial (ECST). Lancet. 1998;351:1379-87.

5. Mayberg MR, Wilson SE, Yatsu F, Weiss DG, Messina L, Hershey LA et al. Carotid endarterectomy and prevention of cerebral ischemia in symptomatic carotid stenosis. JAMA. 1991;266: 3289-94.

6. Hobson RW 2nd, Weiss DG, Fields WS, Goldstone J, Mooew WS, Towne JB et al. Ef?cacy of carotid endarterectomy for asymptomatic carotid stenosis. The Veterans Affairs Cooperative Study Group. N Engl J Med. 1993;328:221-7.

7. Study ECftACA. Endarterectomy for asymptomatic carotid artery stenosis. JAMA. 1995;273:1421-8.

8. República de Colombia. Departamento Administrativo Nacional de Estadística. Censo general 2005. http://www.dane. gov.co/censo/

9. Goldstein LB, Adams R, Alberts MJ, Appel LJ, Brass LM, Bushnell CD, et al. Primary prevention of ischemic stroke. A guideline from the American Heart Association/American Stroke Association Stroke Council: Cosponsored by the Atherosclerotic 
Peripheral Vascular Disease Interdisciplinary Working Group; Cardiovascular Nursing Council; Clinical Cardiology Council; Nutrition, Physical Activity, and Metabolism Council; and the Quality of Care and Outcomes Research Interdisciplinary Working Group. Circulation. 2006;113:e873-923.

10. Hamel M, Henderson WG, Khuri SF, Daley J. Surgical outcomes for patients aged 80 and older: Morbidity and mortality from major noncardiac surgery. J Am Geriatr Soc. 2005;53:424-9.

11. Kazmers A, Perkins AJ, Huber TS, Jacobs LA. Carotid surgery in octogenarians in Veterans Affairs medical centers. J Surg Res. 1999;81: $87-90$.

12. Weintraub WS, Craver JM, Cohen CL, Jones EL, Guyton RA. In?uence of age on results of coronary artery surgery. Circulation. 1991 Nov; 84(5 Suppl): III:226-35.

13. Krumholz HM, Forman DE, Kuntz RE, Balm DS, Wey JY. Coronary revascularization after myocardial infarction in the very elderly: Outcomes and long-term follow-up. Ann Intern Med. 1993;119:1084-90.

14. Glower DD, Christoper TD, Milano CA, White WD, Smith LR, Jones RH et al. Performance status and outcome after coronary artery bypass grafting in persons aged 80-93. Am J Cardiol. 1992;70:567-71.

15. Estes JM, Guadagnoli E, Wolf R, Logerfo FW, Whittemore AD. The impact of cardiac comorbidity after carotid endarterectomy. J Vasc Surg. 1998;28:577-84.
16. Barnett HJ, Taylor DW, Eliasziw M, Fox AJ, Ferguson GG, Haynes RB, et al. Bene?t of carotid endarterectomy in patients with symptomatic moderate or severe stenosis. North American Symptomatic Carotid Endarterectomy Trial Collaborators. $N$ Engl J Med. 1998;339:1415-25.

17. Executive Committee for the Asymptomatic Carotid Atherosclerosis Study. Endarterectomy for asymptomatic carotid artery stenosis. JAMA. 1995;273: $1421-8$

18. Jeanwan L, Chung TK, Lancaster RT, LaMuraglia, Conrad MF, Cambria RP. Outcomes after carotid endarterectomy: Is there a high risk population? A national surgical Quality Improvement Program Report. J Vasc Surg. 2009;49:331-9.

19. Stoner MC, Abbott WM, Wong DR, Hua HT, LaMuraglia GM, Kwolek L, et al. Defining the high-risk patient for carotid endarterectomy: an analysis of the prospective National Surgical Quality Improvement Program database. J Vasc Surg. 2006;43:285-96.

20. Sidawy AN, Zwolak RM, White RA, Siami FS, Schermerhorn ML, Sicard GA, SVS Carotid Vascular Registry: CAS vs. CEA outcomes comparison (2008) Presented at the Annual Meeting of the SVS, San Diego, California, June 4-8.

21. Timaran $\mathrm{CH}$, Rosero EB, Veith FJ, Clagett $\mathrm{JG}$, Valentine RJ, Smith ST, et al. Intracranial hemorrhage after carotid endarterectomy and carotid artery stenting in the United States in 2005 (2008) Presented at the Annual Meeting of the SVS, San Diego, Calif, June 4-8.

22. Matsen SL, Chang DC, Perler BA, Roseborough GS, Williams GM. Trends 
in the in-hospital stroke rate following carotid endarterectomy in California and Maryland. J Vasc Surg. 2006;44:488-95.

23. Sheikh K, Bullock C. Variation and changes in state-specific carotid endarterectomy and 30-day mortality rates, United States, 1991-2000. J Vasc Surg. 2003;38:779-84.

24. Endarterectomy for asymptomatic carotid artery stenosis. Executive Committee for the Asymptomatic Carotid Atherosclerosis Study. JAMA. 1995;273:1459-61.

25. Inzitar D, Eliasiw M, Gates P, Harpe B, Richard KT, Brenda L et al. North American Symptomatic Endarterectomy trial. N Engl J Med. 2000;342:1693-700.

26. Thomas PC, Grigg M. Carotid artery surgery in the octogenarian. Aust $N Z J$ Surg. 1996;66:231-4.

27. O'Hara PJ, Hertzer NR, Mascha EJ, Beven EG, Krajewski LP, Sullivan TM. Carotid endarterectomy in octogenarians: Early results and late outcome. J Vasc Surg. 1998;27:860-71.

28. Rosenthal D, Rudderman R, Jones DH, Clark MD, Stanton PE Jr, Lamis PA et al. Endarterectomy in the octogenarian: Is it appropriate? J Vasc Surg. 1986;3:782-7.
29. Coyle KA, Smith RB III, Salam AA, Dodson TF, Chaikof EL, Lumsden AB. Carotid endarterectomy in the octogenarian. Ann Vasc Surg. 1994;8: 417-20.

30. Fisher ES, Malenka DJ, Solomon NA, Bubolz TA, Whaley FS, Wenberg JE. Risk of carotid endarterectomy in the elderly. Am J Public Health. 1989;79: 1617-20.

31. Hsia DC, Krushat WM, Moscoe LM. Epidemiology of carotid endarterectomies among Medicare beneficiaries. J Vasc Surg. 1992;16:201-8.

32. Cronenwett JL, Birkmeyer JD, Nackman GB, Fillinger MF, Beck FR, Zoulak RM et al. Cost-effectiveness of carotid endarterectomy in asymptomatic patients. J Vasc Surg. 1997;25:298-311.

33. Kasper GC, Lohr JM, Welling RE. Clinical benefit of carotid endarterectomy based on duplex ultrasonography. Vasc Endovascular Surg. 2003;37:323-7.

34. Logason K, Karacagil S, Hårdemark HG, Boström A, Hellberg A, Ljungman C. Carotid artery endarterectomy solely based on duplex scan findings. Vasc Endovascular Surg. 2002;36:9-15. 